\title{
Priority-Mobility Aware Clustering Routing Algorithm for Lifetime Improvement of Dynamic Wireless Sensor Network
}

\author{
Rajiv R. Bhandari ${ }^{1}$, Dr. K. Raja Sekhar ${ }^{2}$ \\ Research Scholar ${ }^{1}$, \\ Department of Computer Science and Engineering ${ }^{1,2}$, \\ K L Education Foundation, \\ Vaddeswaram -522502, Guntur Dist., A.P, India
}

\begin{abstract}
Wireless sensor network with mobility is rapidly evolving and increasing in the recent decade. The cluster and hierarchical routing strategy demonstrates major changes in the lifespan of the network and the scalability. The latency, average energy consumption, packet distribution ratio is highly impacted due to a lack of coordination between cluster head and extreme mobile network nodes. Overall efficiency of highly mobile wireless sensor network is reduced by current techniques such as mobilityconscious media access control, sleep/wakeup scheduling and transmission of real-time services in wireless sensor network. This paper proposes a novel Priority-Mobility Aware Clustering Routing algorithm (p-MACRON) for high delivery of packets by assigning fair weightage to each and every packet of node. To automatically decide the scheduling policy, reinforcement learning approach is integrated. The mixed approach of priority and selflearning results into better utilization of energy. The experimental result shows comparisons of slotted sense multiple access protocol, AODV, MEMAC and P-MACRON, in which proposed algorithm delivered better results in terms of interval, packet size and simulation time.
\end{abstract}

Keywords-Cluster; routing; sleep scheduling; priority; reinforcement

\section{INTRODUCTION}

Recently wireless sensor networks are rapidly growing and shifting their paradigm from static to dynamic. Such dynamic changing environment are highly impacted by traditional MAC protocol and sleep scheduling algorithm. Newly developed mobile sensors are advance and much more constrained related to mobility [1] [2], routing protocol, scheduling and clustering [3], [4], [5]. One of the most important features to consider in fast-growing WSN is node mobility and data transmission in real time. A Preemptive Priority-Based Data Fragmentation Scheme proposed [6] where high priority packets and low priority packets are handled. FROG-MAC focuses on fragmentation of packets so that higher priority packets need not to wait for longer time while low priority packets are transferred. The limitation of this scheme is interference of high priority packets while low priority packets are in transmission. Fasee Ullah et. all [7] proposed TRIP-ECC protocol which mainly classify priority of data into four different categories like usual data, on demand data, emergency data of low threshold reading and high threshold reading. This classification works well with Wireless body network but did not work well in heterogeneous network where real time delivery and fair weightage for all nodes. To handle the mobility and scalability of network Mahdi Zareei et al. [5] [8] has done an extensive survey of mobility aware MAC protocol. Based on their survey the paper categorized MAC protocol Scheme in four major categories: General active/sleep time, Slotted TDMA based MAC protocol, preamble sampling MAC protocol and hybrid MAC protocol. Mobility aware protocol is well described with their pros and cons in this paper. MEMAC algorithm is proposed by Bashir Yahya et al. [9] is a hybrid MAC protocol to handle the mobility of nodes with cluster creation, shift, leave, join operation very well. The main constrain of this protocol is handling mobility in large and scalable network. Dayong ye and Minjie Zhang implemented selfadaptive sleep/wakeup scheduling algorithm. This algorithm avoids use of duty cycling to overcome the problem of packet deliver and energy saving. This algorithm uses the concept of Reinforcement learning to achieve better results and algorithm indeed gives best results. The authors didn't test the algorithm on any of cluster routing protocol. This work we have extended in our P-MACRON.The primary role of proposed algorithm in further section is to handle the traffic in mobile network by allocating fair and equal weightage all node. During the data transmission, the node priority is validated by extracting the details from the Packet Header. The remaining paper is organized as follows the background knowledge is described in Section 2, Section 3 specifies proposed work, implementation of work is mentioned in Section 4, results are discussed in Section 5 followed by conclusion in Section 6 .

\section{BACKGROUND KNOWLEDGE}

The proposed work is extension of mobility aware clustering routing MACRON algorithm proposed in [10] [11]. This algorithm wisely chooses the cluster head and its members based on probability distribution functions and iterative calculation. Unlike conventional algorithms, MACRON manages node mobility effectively by performing leave join operations iteratively. The MACRON algorithm operates in three phases: 1) Network creation MACRON Algorithm, 2) Self-healing Scheduling MACRON Algorithm, 3) Self-healing scheduling approach using Reinforcement Learning. In this section, these three phases are described in detailed. 


\section{A. Network Creation}

The system consists of base station and sensor nodes. The base station initiated clustering process based on node deployment and coverage area. Clustering is executed by an iterative process with probability distribution function. The initial probability of each node is determined based on the one-hop connectivity distance to reach all the covered sensor nodes. By applying LEACH method with energy parameter the updated probability for each node is calculated. Normalize the estimated probability by computing the ratio of the current probability value to the cumulative probability value of all sensors [1] [10]. The node with max probability is selected as Cluster head. The node with one hop distance are selected as members for cluster head. The same process will continue until all nodes in the network are reached to the end [12], [13]

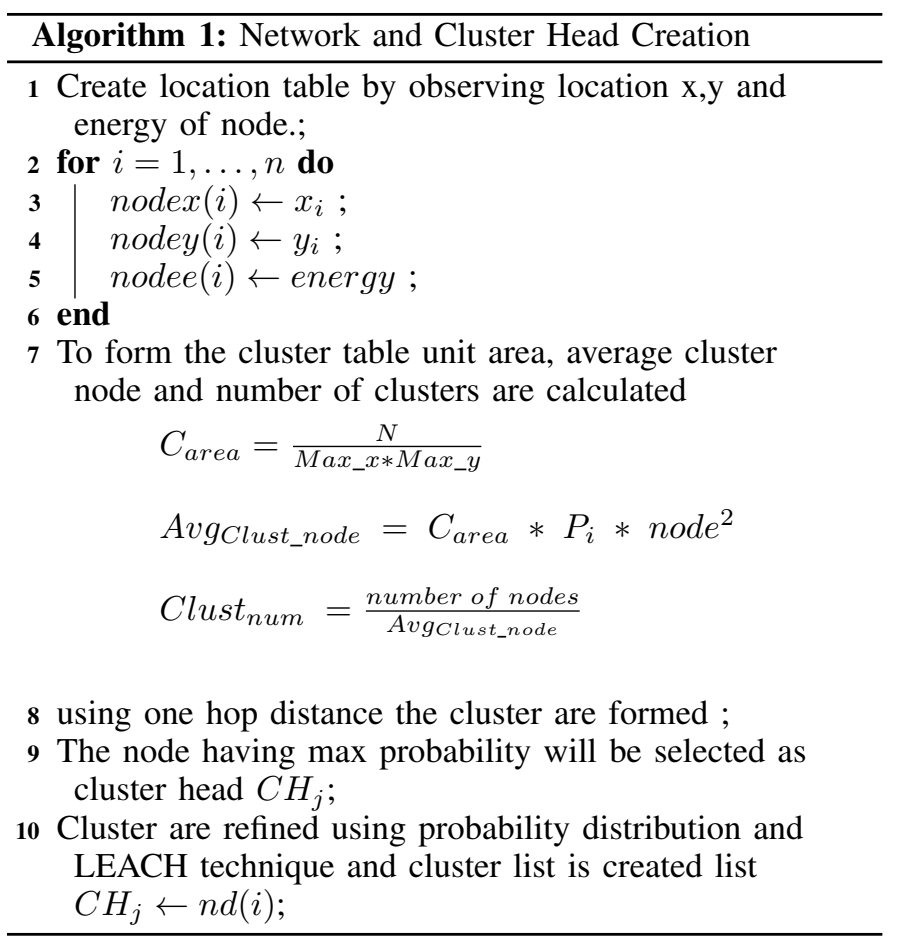

\section{B. Algorithm: Join, Shift and Leave Operation}

Cluster member chooses tentative cluster head based on shortest reachability and sends leave message to old cluster head and join message to newly elected cluster head. If one cluster head receives $\mathrm{CH}$ announcement from other overlapping cluster head, then need to find cluster probability. The node with the greater probability retains the head position so the lower probability $\mathrm{CH}$ performs the cluster shifting process by sending the shifting message to both $\mathrm{CM}$ and overlapping $\mathrm{CH}$, then cluster head assigns slot to members [14] [15]

\section{Self Healing Scheduling Approach using Reinforcement Learning}

Self-healing scheduling approach using Q-learning algorithm decides when to transmit the packets in sub timeslot. This algorithm works with rewards and penalty so that

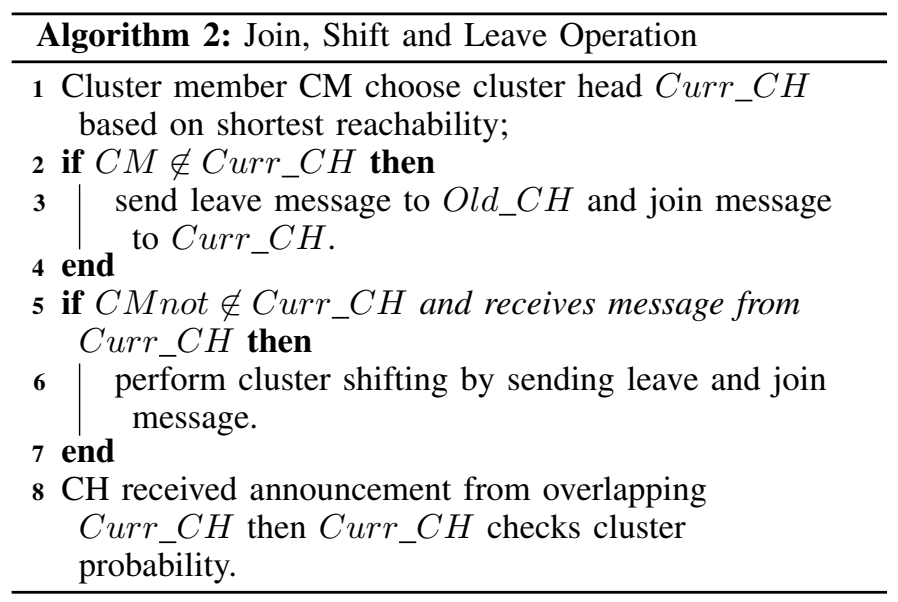

all nodes will select transmission of packets with proper prediction in future. This algorithm works with sleep and active states by adjusting the idle state of node as it consumes lot of energy[3][16]. Initially the learning rate $\zeta$, and $\delta$ are determined by observing the how much amount of prior information will be override by the recent set of actions. The set of actions considered here is $a \in\{$ transmit,listen, sleep $\}$

Generally it takes the value between $[0,1]$, Here, 0 means that the prior information will be retained as it is and 1 indicates that it will positively identify the prior information. The discount factor $\gamma$ also takes the value between $[0,1]$, the value nearer to 0 indicates the node is naive and it deals with the recent actions only and, the discount factor value approaching to 1 indicates that the node is promising with high reward value.

For reinforcement learning approach, the policy defined in [17] [18] is adopted. By considering this policies the payoff, average payoff will be decided and the transmission slot will be normalized.

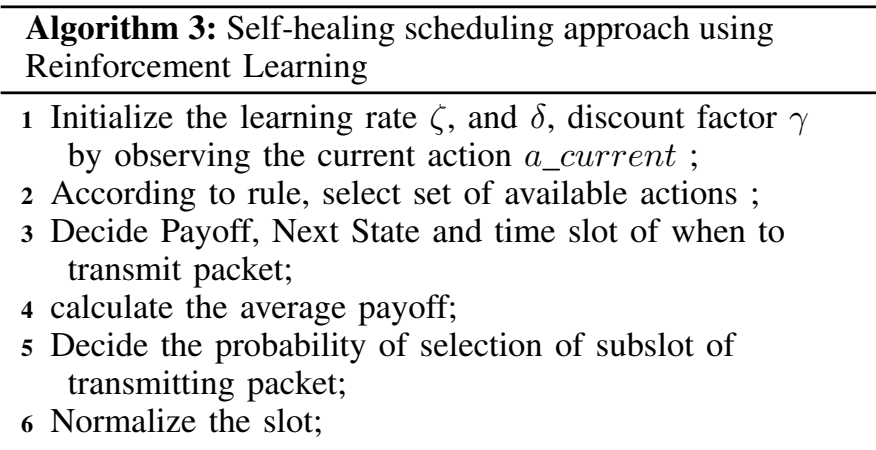

\section{PROPOSED WORK}

The proposed work is demonstrated in three sections. The detail architecture is shown in Section 1, Section 2 focuses on how reinforcement learning is integrated in proposed work. The $\mathrm{P}-\mathrm{MACRON}$ algorithm is described in Section 3.

\section{A. Architecture of Proposed Algorithm}

The Architecture of sensor network shown in Fig. 1 is created by consideration of Node 0 as Base node and base 
station initiate the clustering process on nodes deployment and coverage area of sensors. The network will perform operations such as joining another network, switching from one network to another and finding nodes in the network about to die. The node with the greater probability retains the head position so the lower probability $\mathrm{CH}$ performs the cluster shifting process by sending the shifting message to both $\mathrm{CM}$ and overlapping $\mathrm{CH}[19]$.

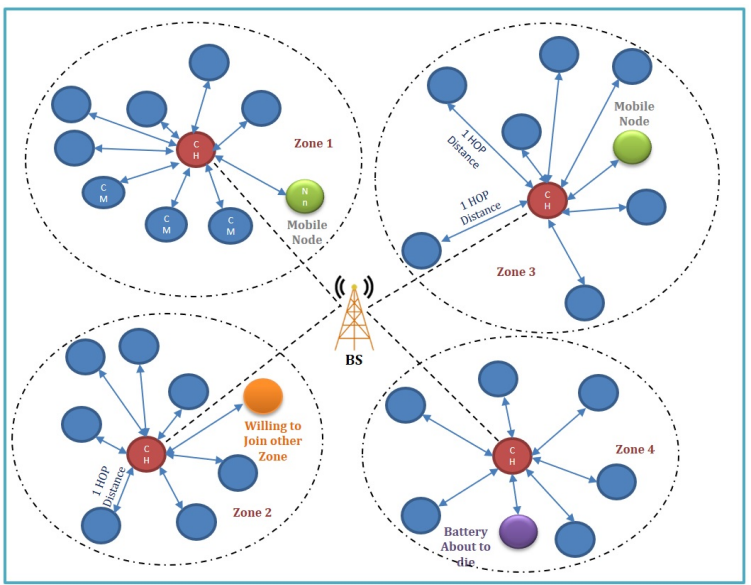

Fig. 1: System Architecture with Join, Leave, Shift Operation.

\section{B. Reinforcement Learning Integration with Proposed Algo- rithm}

Reinforcement learning enables the nodes to learn best possible actions in order to take best decision with previous experiences as shown in figure 2.

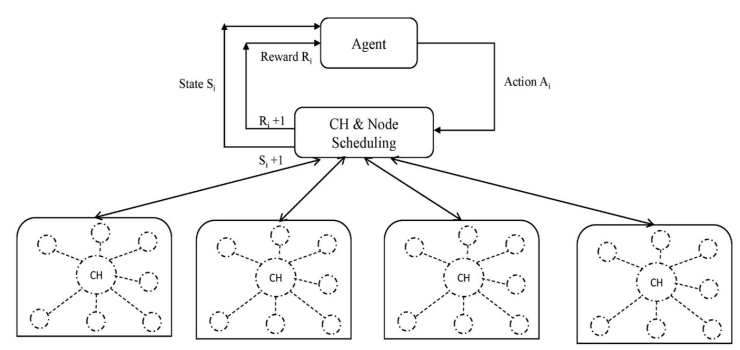

Fig. 2: Self-healing Sleep/wakeup Structure using Reinforcement Learning Algorithm.

\section{P-MACRON Algorithm}

The proposed work contributes in maximization of network lifetime for dynamic wireless sensor network and giving fair chance to all nodes in the network to transmit the data. The three objectives of algorithm are:

- $\quad$ MACRON algorithm for minimum energy consumption that works effectively and proficiently in dynamic mobile wireless area network.
- Self-healing scheduling using reinforcement learning approach for long life of wireless sensor network.

- A new priority based hybrid approach for improving lifetime of Wireless Sensor Network to deliver realtime data by assigning fair weightage to every node.

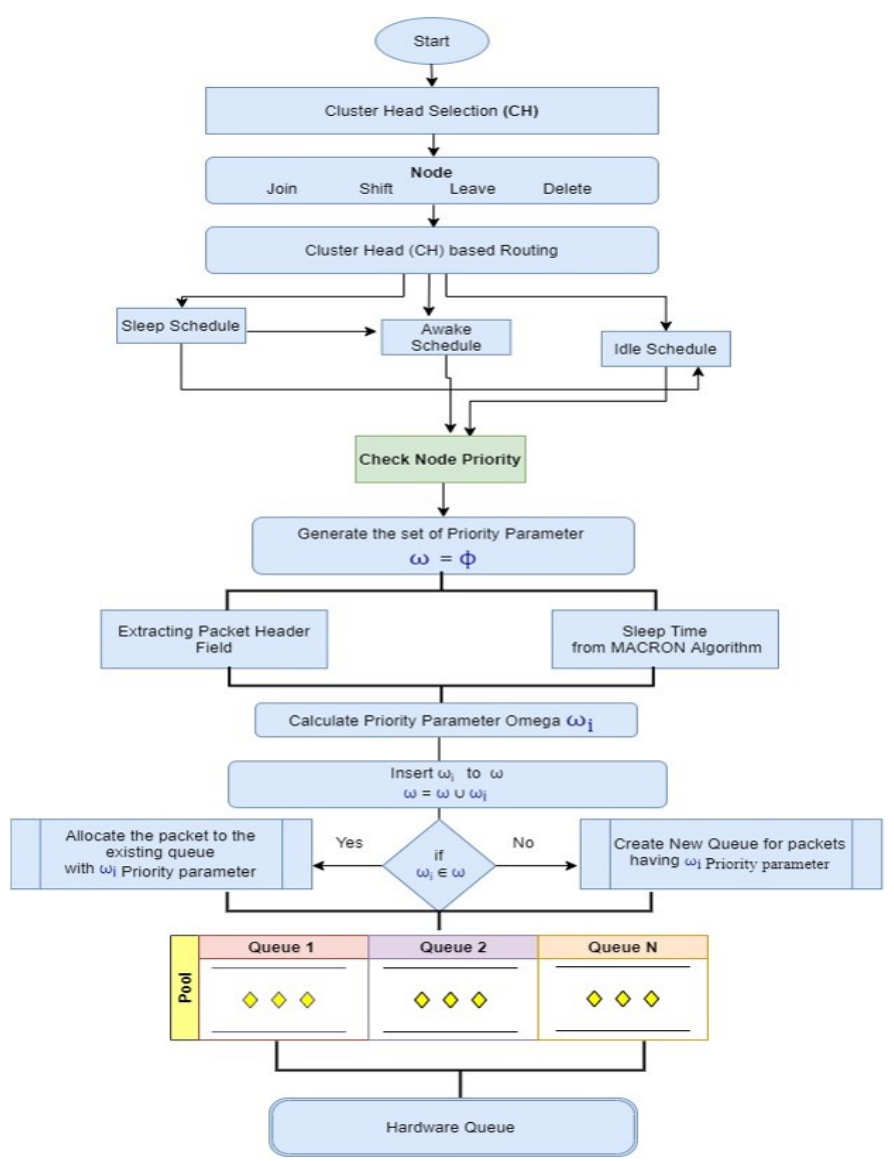

Fig. 3: Flow of Priority-MACRON Algorithm.

The third objective of the algorithm is proposed here to elongate the lifetime of wireless sensor network. A main research theme in dynamic wireless sensor networks is the nature of real-time data transmission and equal chance for every node. P-MACRON maintains priority queue $[P Q 1, P Q 2, P Q 3 \ldots P Q n]$ and tuning factor $\omega$ to assign fair weightage to all nodes. Proposed P-MACRON extract packet header parameters and consider scheduling value like sleep and active. Self-healing scheduling algorithm adjust their idle state with sleep and active state this will help in fast transmission of packets. Each node maintaining its sleep and active state and communicate its schedule with cluster head locally. All cluster heads will dynamically maintain their cluster table with schedules communicated by nodes.During the data transmission, the node priority is validated by extracting the details from the Packet Header. The packet header contains the information about the parameters such as the average number of nodes distributed in the clusters, number of packets in the buffer, sleep time for each sensor, sensors connectivity with its neighbors and the mobility of the sensors [20] [21]. The weighted fair queuing model is applied to decide the priority based on the uniformly distributed weight values. The cumulative value of 
the weight is maintained to the unit value and the weighted value is computed for all parameters related to the priority value. The weighted priority value of the data packet is used to decide the packet queue which is used to buffer the packet. If the priority is not available in the queue then it is included in the queue pool to complete the data transmission. If the priority is available in the queue then the same pool is used to complete the data transmission. Based on the selected queue pool the transmission priority is assigned to each packet. In the Mac layer, the packet transmission is performed based on the assigned priority. Fig. 3 is explaining the overall flow of P-MACRON Algorithm.

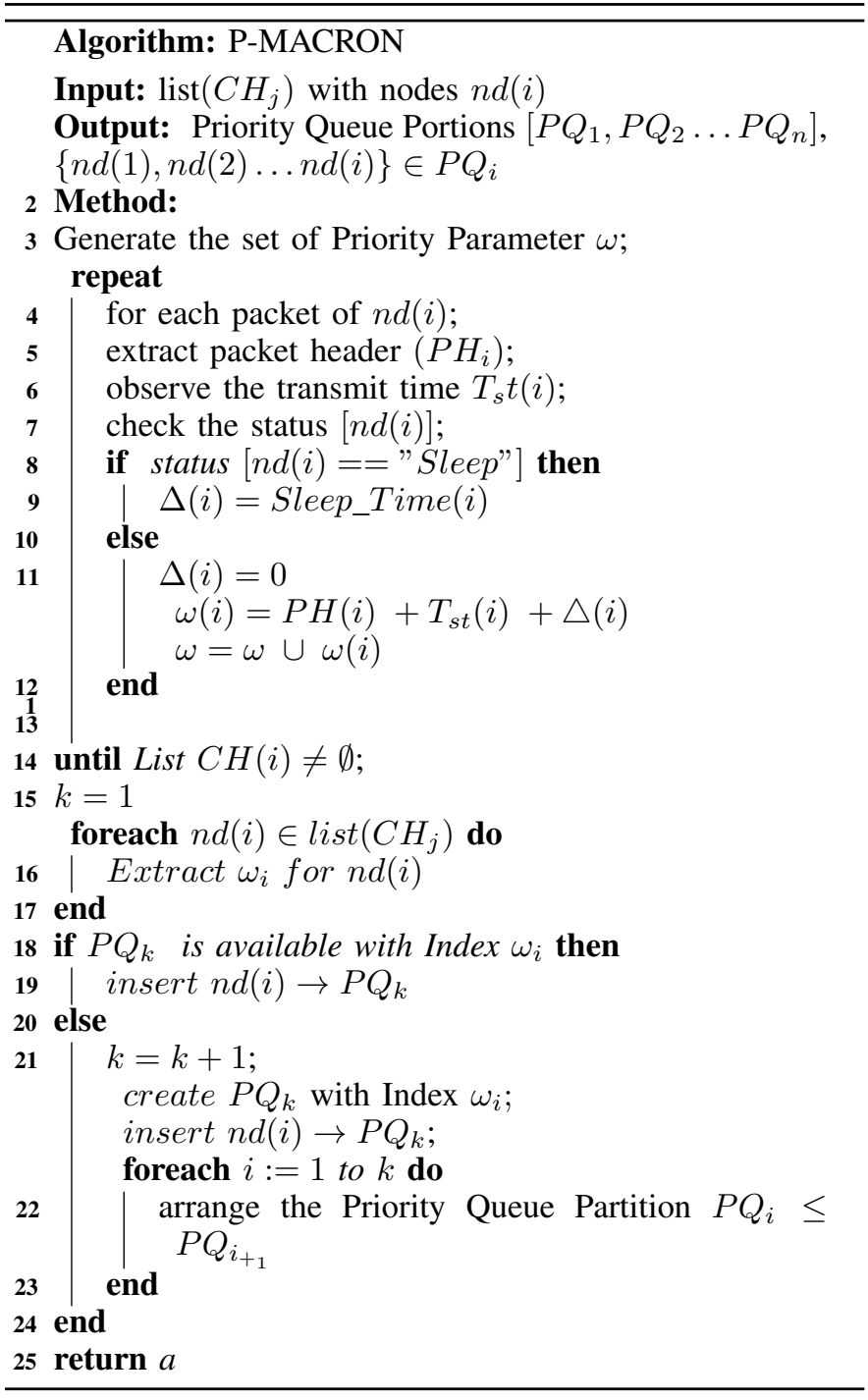

\section{EnVIRONMENT SETUP AND Results}

This section proposed more stable and consistent result over slotted sense multiple access algorithm. The framework uses NS2.34 to conduct performance study to analyses PMACRON and SSMA efficiency [22], and to evaluate PMACRON's feasibility. The network size of 500 by 500 square meter is considered and 101 nodes are randomly deployed where node 0 is working as Base station. The simulation results
TABLE I: Simulation Parameters

\begin{tabular}{|c|c|c|c|c|}
\hline Particular & AODV & MEMAC & SSMA & P-MACRON \\
\hline Node & 101 & 101 & 101 & 101 \\
\hline Network Size & \multicolumn{4}{|c|}{$500 * 500$ sq mtr } \\
\hline MAC & AODV & MEMAC & SSMA & 802.11 \\
\hline Radio Range & \multicolumn{4}{|c|}{$3.652 e-80$ s } \\
\hline Simulation Time & \multicolumn{4}{|c|}{ Sense Traffic } \\
\hline Traffic Source & \multicolumn{4}{|c}{ bytes } \\
\hline Packet Size & \multicolumn{4}{|c}{ Random Mobility } \\
\hline Mobility Model & \multicolumn{4}{c}{} \\
\hline
\end{tabular}

of P-MACRON in comparison with SSMA are based on three main parameters like interval, packet size and simulation time. The simulation parameters are mentioned in table I.

Fig. 4 and Fig. 5 are showing comparison of P-MACRON with SSMA, MEMAC, AODV. Some of the observation for the performance of the algorithm are as follows:

- The most significant efficiency metric for wireless sensor networks is energy consumption. The average energy consumption for AODV, MEMAC, SSMA under variable interval and packet size for mobile network is shown figure. P-MACRON outperforms AODV, MEMAC and SSMA as mobility increases.

- $\quad$ The percentage of packet delivery of P-MACRON to base station is significantly promising over AODV, MEMAC and SSMA.

- The interval and packet size changes from 0.1000 to 0.2000 and 20 to 40 respectively. Under the mobile network scenario, the AODV, MEMAC have high delay but still SSMA and P-MACRON shows better performance. If we compare SSMA, MEMAC and AODV [23] as PMACRON adopts a clustering

- $\quad$ approach with one hop distance, the hop count needed to reach the destination is significantly fine.

- The Overall lifetime of network is main focus of proposed algorithm. Figure shows significant growth in improving lifetime of network over SSMA, MEMAC and base case AODV.

- The number of packets per second received at receiver is defined as throughput. The number of packets delivered at receiver end is quite good in P-MACRON and MEMAC over SSMA and AODV.

\section{CONClusion AND Future Work}

Using the clustering and scheduling algorithm, assigning priorities has been a primary concern of the industrial sector and the defense sector. P-MACRON algorithm mainly focuses on assigning fair weightage to all packets of nodes for reliable and on time delivery of packets. As observed, P-MACRON lays the groundwork to group nodes at one hop distance into clusters with priority leading to energy-efficient routing and scheduling using machine learning algorithm has proven to be one of the most effective approaches for exclusive dynamic wireless sensor networks. P-MACRON algorithm works efficiently for extensively dynamic algorithm with distinguish method of cluster creation and self-healing scheduling algorithm using Q Learning Algorithm. Results from simulation 


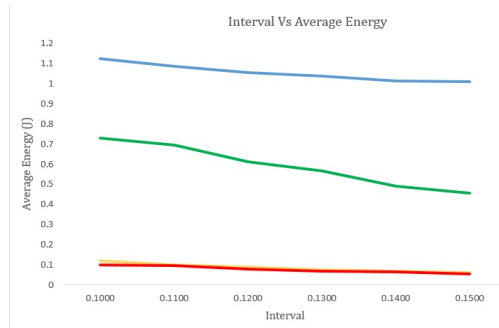

(a) Interval Vs Average Energy

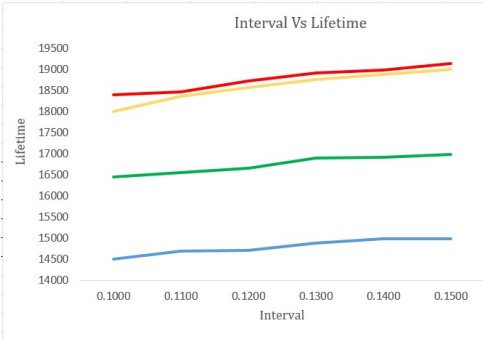

(d) Interval Vs Lifetime

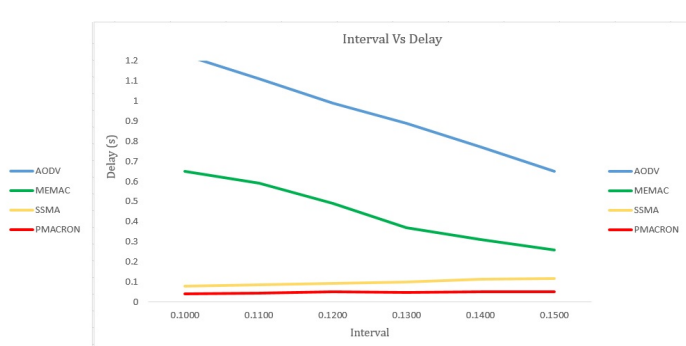

(b) Interval Vs Delay

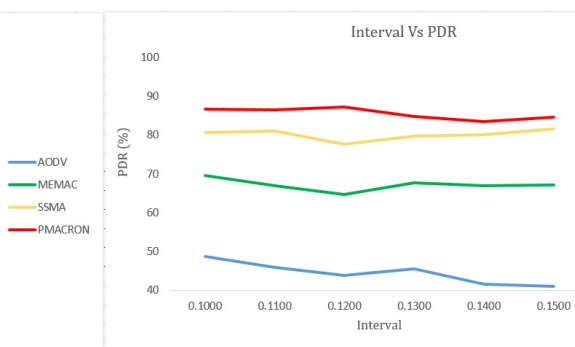

(e) Interval Vs PDR

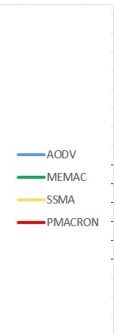

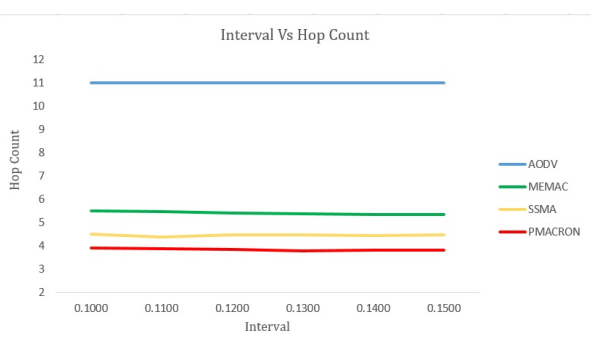

(c) Interval Vs Hop Count

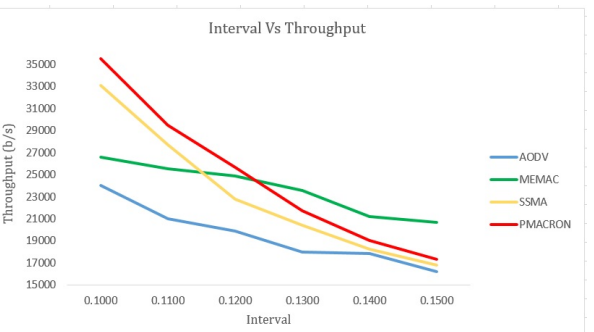

(f) Interval Vs Throughput

Fig. 4: Comparative Analysis based on Interval.

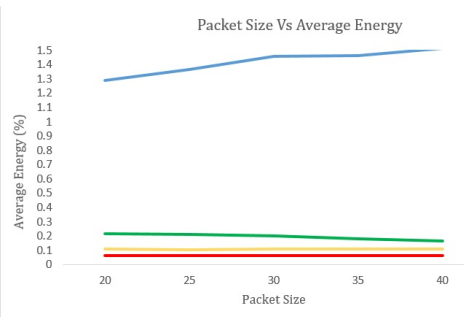

(a) Packet Size Vs Average Energy

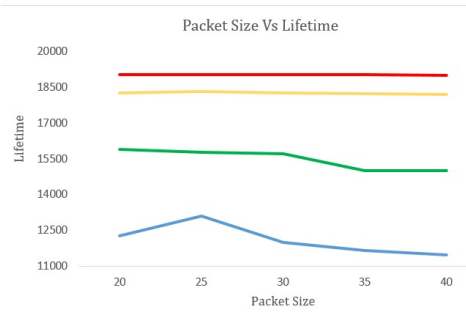

(d) Packet Size Vs Lifetime
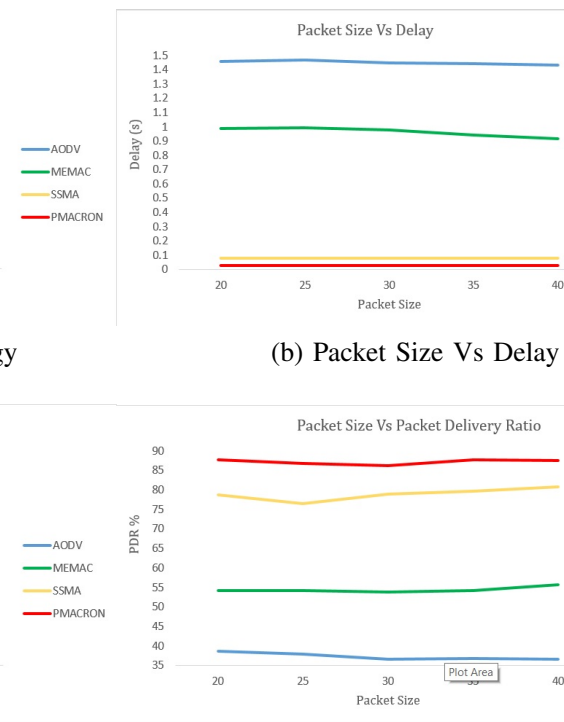

(e) Packet Size Vs PDR
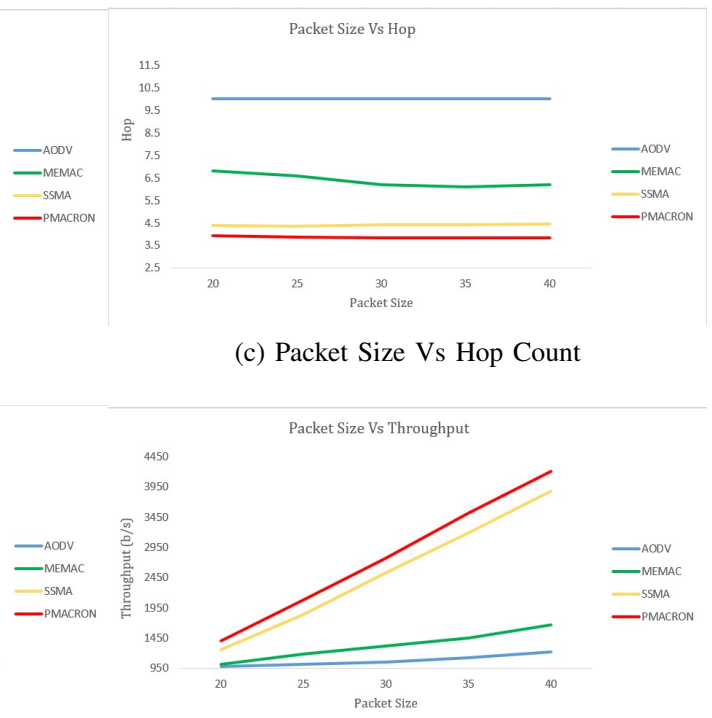

(f) Packet Size Vs Throughput

Fig. 5: Comparative Analysis based on Packet Size.

reveal that P-MACRON performs much better in terms of Average Energy, delay, hop count, packet delivery ration and throughput than the SSMA, MEMAC and AODV algorithms to achieve high lifetime with high mobility. In future, we are planning to implement P-MACRON with topology independent network with more parameter such as multihop transmission, collision.

\section{REFERENCES}

[1] B. Yahya and J. Ben-Othman, "An adaptive mobility aware and energy efficient mac protocol for wireless sensor networks," in 2009 IEEE
Symposium on Computers and Communications. IEEE, 2009, pp. 1521.

[2] M. Anusha and S. Vemuru, "Cognitive radio networks: State of research domain in next-generation wireless networks-an analytical analysis," in Information and Communication Technology for Sustainable Development. Springer, 2018, pp. 291-301.

[3] D. Ye and M. Zhang, "A self-adaptive sleep/wake-up scheduling approach for wireless sensor networks," IEEE transactions on cybernetics, vol. 48, no. 3, pp. 979-992, 2017.

[4] E. Srie Vidhya Janani and P. Ganesh Kumar, "Energy efficient cluster based scheduling scheme for wireless sensor networks," The Scientific World Journal, vol. 2015, 2015. 
[5] M. Zareei, A. M. Islam, C. Vargas-Rosales, N. Mansoor, S. Goudarzi, and M. H. Rehmani, "Mobility-aware medium access control protocols for wireless sensor networks: A survey," Journal of Network and Computer Applications, vol. 104, pp. 21-37, 2018.

[6] A. A. Khan, S. Ghani, and S. Siddiqui, "A preemptive priority-based data fragmentation scheme for heterogeneous traffic in wireless sensor networks," Sensors, vol. 18, no. 12, p. 4473, 2018.

[7] F. Ullah, Z. Ullah, S. Ahmad, I. U. Islam, S. U. Rehman, and J. Iqbal, "Traffic priority based delay-aware and energy efficient path allocation routing protocol for wireless body area network," Journal of Ambient Intelligence and Humanized Computing, vol. 10, no. 10, pp. 3775-3794, 2019.

[8] P. Bansal, P. Kundu, and P. Kaur, "Comparison of leach and pegasis hierarchical routing protocols in wireless sensor networks," International Journal on Recent Trends in Engineering \& Technology, vol. 11, no. 1, p. 139, 2014.

[9] L. Karim, N. Nasser, T. Taleb, and A. Alqallaf, "An efficient priority packet scheduling algorithm for wireless sensor network," in 2012 IEEE international conference on communications (ICC). IEEE, 2012, pp. 334-338.

[10] R. R. Bhandari and K. Rajasekhar, "Study on improving the network life time maximazation for wireless sensor network using cross layer approach," International Journal of Electrical and Computer Engineering, vol. 6, no. 6, p. 3080, 2016.

[11] M. El Ouadi and A. Hasbi, "Comparison of leach and pegasis hierarchical routing protocols in wsn," 2020.

[12] L. Farhan and R. Kharel, "Internet of things scalability: communications and data management," in Modern Sensing Technologies. Springer, 2019, pp. 311-329.

[13] B. Jan, H. Farman, H. Javed, B. Montrucchio, M. Khan, and S. Ali, "Energy efficient hierarchical clustering approaches in wireless sensor networks: A survey," Wireless Communications and Mobile Computing, vol. 2017, 2017.

[14] A. Lohachab, A. Lohachab, and A. Jangra, "A comprehensive survey of prominent cryptographic aspects for securing communication in postquantum iot networks," Internet of Things, vol. 9, p. 100174, 2020.

[15] S. Hamad, K. Alheeti, Y. Ali, and S. Shaker, "Clustering and analysis of dynamic ad hoc network nodes movement based on fcm algorithm," 2020.

[16] H. Mohammad and A. C. Sastry, "Acnm: Advance coupling network model sleep/awake mechanism for wireless sensor networks," International Journal of Engineering \& Technology, vol. 7, no. 1.1, pp. 350354, 2018.

[17] R. R. Bhandari and K. Rajasekhar, "Mobility aware clustering routing algorithm (macron) to improve lifetime of wireless sensor network," International Journal of Recent Technology and Engineering (IJRTE), vol. 8, no. 2, p. 76, 2019.

[18] N. Srikanth and M. S. Prasad, "Energy efficient trust node based routing protocol (eetrp) to maximize the lifetime of wireless sensor networks in plateaus," International Journal of Online and Biomedical Engineering (iJOE), vol. 15, no. 06, pp. 113-130, 2019.

[19] R. R. Bhandari and K. Rajasekhar, "Energy-efficient routing-based clustering approaches and sleep scheduling algorithm for network," Inventive Communication and Computational Technologies: Proceedings of ICICCT 2019, vol. 89, p. 293, 2019.

[20] P. Goswami, Z. Yan, A. Mukherjee, L. Yang, S. Routray, and G. Palai, "An energy efficient clustering using firefly and hml for optical wireless sensor network," Optik, vol. 182, pp. $181-185,2019$. [Online]. Available: http://www.sciencedirect.com/science/article/pii/S0030402618320540

[21] K. R. Nirmal and K. Satyanarayana, "Map reduce based removing dependency on $\mathrm{k}$ and initial centroid selection mr-redic algorithm for clustering of mixed data," measurement, vol. 5, p. 6.

[22] H. Oh and C. T. Ngo, "A slotted sense multiple access protocol for timely and reliable data transmission in dynamic wireless sensor networks," IEEE Sensors Journal, vol. 18, no. 5, pp. 2184-2194, 2018.

[23] S. K. A. AYUSHREE, "Comparative analysis of aodv and dsdv using machine learning approach in manet," Journal of Engineering Science and Technology, vol. 12, no. 12, pp. 3315-3328, 2017. 